\title{
Tibial Insertion of Patellar Tendon: Arthroscopic Approach
}

\section{Marco Martins Lages ${ }^{1 *}$ and Max Rogerio Freitas Ramos ${ }^{2}$}

${ }^{1}$ Servico de Ortopedia e Traumatologia Prof. Nova Monteiro, Hospital Municipal

Miguel Couto, Brazil

${ }^{2}$ Hospital Universitário Gafrée-Guinle, UNIRIO, Brazil

*Corresponding Author: Marco Martins Lages, Servico de Ortopedia e

Traumatologia Prof. Nova Monteiro, Hospital Municipal Miguel Couto, Brazil.
Received: February 06, 2021

Published: February 26, 2021

(C) All rights are reserved by Marco Martins

Lages and Max Rogerio Freitas Ramos.

\begin{abstract}
Osgood-Schlatter's disease is responsible for a significant portion pain in adolescent knee. Conservative treatment is successful in more than $90 \%$ of cases. In unresolved cases, surgery is recommended for tendon debridement and resection of the remaining ossicle. Arthroscopy is a valid surgical procedure in these cases.
\end{abstract}

Keywords: Osgood-Schlatter; Arthroscopy

\section{Introduction}

Osgood-Schlater's disease was described in 1903 by doctors Robert Bayley Osgood [1] and Carl Schlatter [2] as an inflammatory process in the apophysis of tibial tuberosity in adolescents, affecting up to $20 \%$ of active population of this age group [3].

Etiopathogenesis involves excessive and continuous traction of patellar tendon at its distal insertion, by a powerful action of quadriceps muscle in activities such as soccer and running during growth spurt phase.

More than $90 \%$ of cases can be treated conservatively, with low risk of complications. In some cases, an intrasubstantial calcification of the distal insertion of patellar tendon may remain, disturbing the region when kneeling and even limiting some physical activities [4].

In these cases, when conservative treatment is unsuccessful, ranging from 5 to $12 \%[12,15,19,30]$, ossification resection is indicated, always trying to preserve vascularity and integrity of distal tendon insertion. As this is not a disabling pain, it is common for the patient to wait, on average, up to 3 years until taking the decision for surgical treatment $[12,28]$.

Surgery, when indicated, should be performed after full skeletal maturity. Approximately $75 \%$ of patients return to sports activities at the same pre-injury level, without any complications. Only 2 to $6 \%$ of patients reported dissatisfaction with surgical treatment $[6,10]$.

Rarely, in younger patients, prophylactic percutaneous fixation may be indicated in those who have not yet completely closed physis [29,32]. The removal of the synthesis after one year of surgery in these cases is mandatory.

Klein [8] was the first to describe an arthroscopic technique, through the enlarged inferior-lateral and inferior-medial parapatellar portals, allowing visualization of anterior range through the arthroscopic route. Staying anterior to anterior meniscal horns, it is possible to perform an aggressive debridement of anterior tibial 
slope with aid of a bone shaver, in addition of removing free bodies with grasper forceps [5].

Lui [25] suggested an alteration to this technique, using portals even closer to tibial tuberosity, with a careful regard to the location of the scar, away from the actual injury site, since proximity of both sites may result in painful hypertrophic scarring. A medial and distal portal to the deformity can facilitate the entry of instruments that will remove the ossicle in such cases.

Entry points are located at the same longitudinal position as classic antero-lateral and antero-medial portals, $1 \mathrm{~cm}$ lateral and medial of the patellar tendon, but between 2 and $4 \mathrm{~cm}$ more distal to the original entry point of these portals, regularly used to approach the menisci and the intercondylar region [15]. It starts by lateral portal, and after crossing the anterior interval, the arthroscope light guides the path of medial entry point. Knee is placed in extension position during the procedure and can be flexed to a maximum of $15^{\circ}$. A $70^{\circ}$ arthroscope can facilitate visualization of the intratendinous ossicle.

After procedure, physiotherapy begins on the first postoperative day, with work on range of motion and full load, with a greater focus on reducing edema and strengthening the knee's extension mechanism.

The biggest advantage of this technique is to make longitudinal incision of the tendon unnecessary, reducing morbidity and allowing a quicker return to sports activities, around six weeks. Arthroscopic approach also allows a better posterior view of the ossicles than open lateral parapatellar access [7].

\section{Materials and Methods}

An observational, descriptive study on an analytical basis, compared six different portals for knee arthroscopy, and its visualization of the patellar tendon's tibial insertion, through two different cadaveric specimens at our Anatomic Laboratory of Hospital Universitário Gafrée-Guinle, in Rio de Janeiro, Brazil. A systematic review in literature was also performed, searching key terms related to Osgood-Schlatter disease complications.

Two cadaveric specimens were selected for the knee portals analysis, using a $4 \mathrm{~mm}$ optic with $30^{\circ}$ angle view.
The ease of visualization of patellar tendon's tibial insertion was evaluated, considering the presence of Hoffa's fat, and all bony stops from patellar and femoral joint surfaces, comparing range of field of view between lateral and medial suprapatellar portals, lateral and medial infra-patellar and the portals described by KLEIN 2 $\mathrm{cm}$ distal to the classic infrapatellar portals (Figure 1). It is recommended to keep knee in full extension to $15^{\circ}$ flexion so the tendon is relaxed and the joint space is expanded $[7,8,27]$.

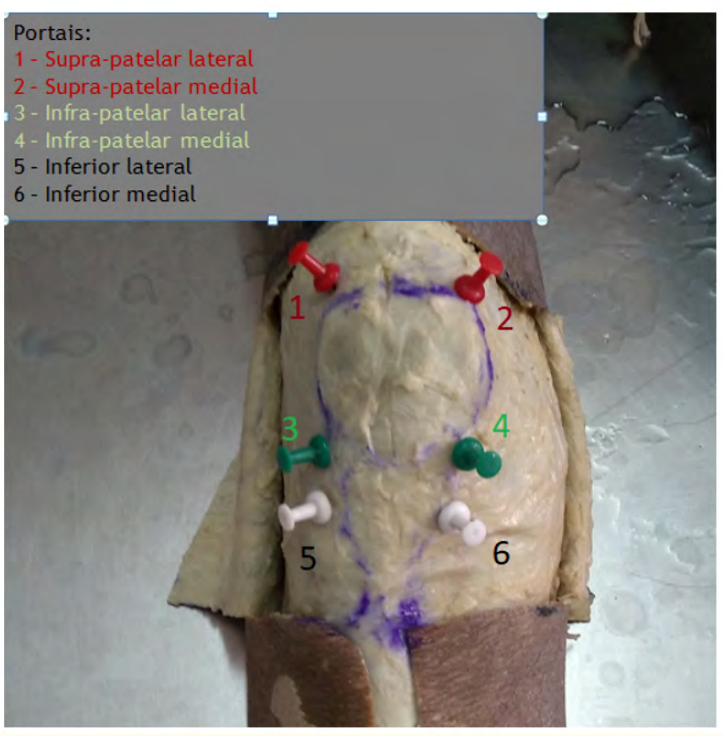

Figure 1: Portals.

Aspects such as patient satisfaction, time to return to sports or work activities, ability to return to the same pre-injury level and complications according to literature were taken into account, which were compared between the open surgery and arthroscopic routes for treatment of this condition.

The following keyterms were searched: OSGOOD-SCHLATTER plus SURGICAL TREATMENT plus/or ARTHROSCOPY plus/or BURSOSCOPY plus/or UNRESOLVED plus/or RESIDUAL plus/or CRONICAL PAIN.

\section{Results}

The patellar tendon insertion was better observed through the lower arthroscopic portals described by LUI ${ }^{28}$. The classic lateral 
infrapatellar portal has a greater struggle as the patellar side wall acts as a stop preventing a better angle to reach the most lateral portion of the tendon.

In the lateral supra-patellar portal, the structure that hides our aim's view was the lateral femoral condyle, which is usually more anterior than the medial condyle. Likewise, the classic medial infrapatellar portal also faces struggles due to the medial wall of the patella.

Surprisingly, the medial supra-patellar portal provided a better viewing angle than the classic infra-patellar ones and can serve as an option as a third accessory portal, in those cases where it is desired to preserve more of Hoffa's fat pad, when an assistant can remove this tissue with a probe through the lower lateral portal, while the camera would enter through this medial suprapatellar portal.

Images related to vision angle of the two best portals (medial supra-patellar and lateral inferior) are showed in figure 2 and 3.

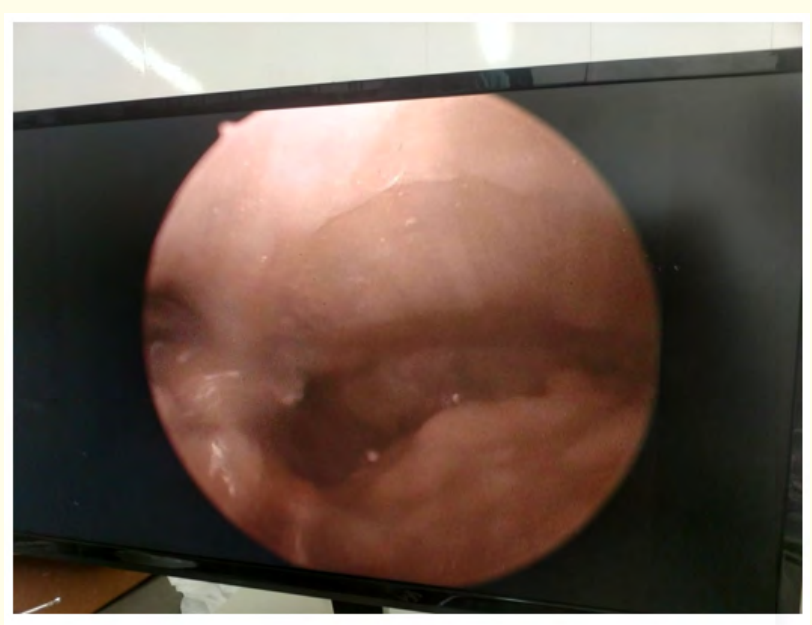

Figure 2: Lateral inferior portal view (portal 5).

Sixteen publications were found talking about surgical treatment of unresolved Osgood-Schlatter disease, with 289 cases reported to the open surgical technique and 42 cases reporting the arthroscopic approach (Table 1).

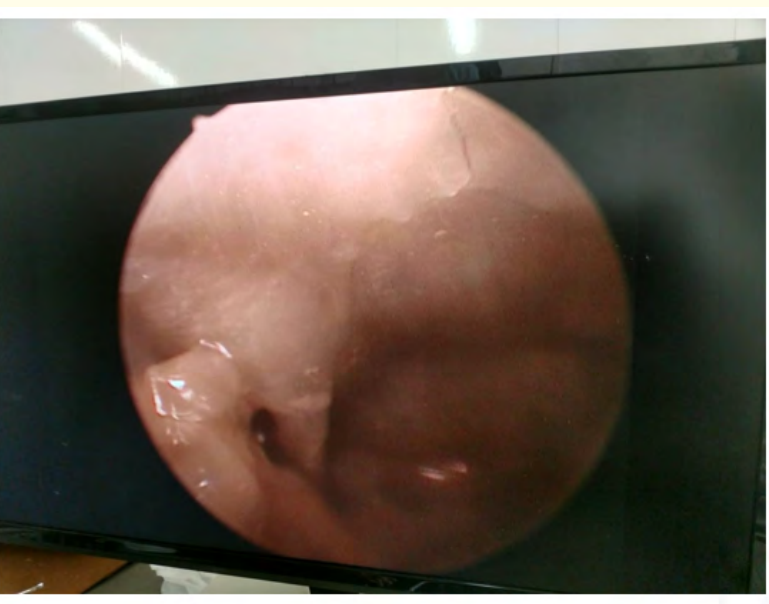

Figure 3: Medial inferior portal view (Portal 6).

\begin{tabular}{|l|c|c|c|}
\hline \multicolumn{1}{|c|}{ Article } & Technique & $\begin{array}{c}\text { Number } \\
\text { of cases }\end{array}$ & Satisfaction \\
\hline Figueiredo & Open & 2 & $100 \%$ \\
\hline Philajamaki & Open & 117 & $99 \%$ \\
\hline Eun & Arthroscopy & 18 & $94 \%$ \\
\hline $\begin{array}{l}\text { Flowers } \\
\text { Bhadreshwar }\end{array}$ & Open & 35 & $95 \%$ \\
\hline Kujala & Open & 14 & $93 \%$ \\
\hline Orava & Open & 62 & $96 \%$ \\
\hline Wysokinska & Arthroscopy & 11 & $91 \%$ \\
\hline El-Husseini & Open & 37 & 974 \\
\hline Beyzadeoglu & Arthroscopy & 11 & $100 \%$ \\
\hline Lee & Arthroscopy & 1 & $100 \%$ \\
\hline Zhi-Yao & Arthroscopy & 1 & $100 \%$ \\
\hline Nierember & Open & 22 & $91 \%$ \\
\hline Choi & Arthroscopy & 1 & $100 \%$ \\
\hline Total & Open & 43 & $95 \%$ \\
\hline Total & Arthroscopy & 289 & $98 \%$ \\
\hline
\end{tabular}

Table 1: Patients submitted to surgery for unresolved Osgood-Schlatter disease. 
The number of cases reported through the arthroscopic approach is still much inferior than the traditional open surgery. Several articles are only one single case report, making scientific experience in literature still very limited. However, some data can already be analyzed: Return to sport practice seems to be smaller through arthroscopic approach, with time off-practice varying from three to six weeks $[7,15,23,24,31]$. Open surgery has a greater average time to return, from six to twelve weeks $[10,13,16,17]$. Only two cases manage to have a fast recovery through the open approach, with only four weeks off-practice.

\section{Discussion}

Although most patients with Osgood-Schlatter's disease have good resolution without the need of any surgery, some cases eventually require surgical treatment to perform the ossicle resection or the osteoplasty of the tibial tuberosity.

Conservative treatment includes rest, especially in the adolescent's acute phase, ice packs, non-steroidal anti-inflammatory drugs, physical therapy with resource of anti-inflammatory devices, such as ultrasound and light amplification by radioisotope emission (LASER), the prescription of eccentric strengthening and stretching exercises of the knee's extensor mechanism, in addition to the use of infrapatellar braces [20]. Corticosteroid infiltration is prohibited, due to the risk of complications with subcutaneous atrophy and myotendinous weakness.

Since this is an area with little soft tissue coverage, there are many different risks from a surgical procedure in this region, such as failures in healing, like keloids and skin necrosis, as well as a possible weakening of the tendon insertion caused by aggressive debridement, of muscle tissue and bone insertion, which may lead to tendon rupture.

Therefore, it is important to minimize those risks beyond the main goal of surgery, which is the pain resolution. With this purpose, there are many different techniques described, as:

- Microperfuration of the tibial tuberosity, aiming to stimulate local neovascularization and better healing, despite the success of this technique is still questionable. GLYNN [9] compared this technique with the classical tubercleplasty, with 22 patients in each group, and it was demonstrated that the osteotomy had way superior results: $77 \%$ of satisfied pa- tients against only $36 \%$ of microperfuration with good results.

- Open surgery through patellar tendon, splitting its fibers longitudinal, allowing a better view of ossicles, although it brings grater damage to tendinous tissue, besides some risk of skin healing complications [14].

- Open surgery through both sides of patellar tendon, which preserves tissue integrity, but with a worst view of intratendinous ossicles and providing limited access to tuberosity deformity [14].

- Endoscopic surgery, which has less tissue damage than open surgery, but with the same limited view of intratendinous ossicles and tuberosity deformity. In such cases, the chisel can be substituted by a bone shaver, allowing even better and harmonic osteoplasty.

EUN [15] described good results through arthroscopic approach, but some view limitations of the tuberosity were related. Indeed, $33 \%$ of patients reported no visual difference between before and after surgery, despite satisfaction of clinical symptoms.

Nierenberg [10] described a fluxogram for Osgood-Schlatter treatment (Figure 4). However, there is still no consensus for which approach is more appropriate as surgical treatment.

Circi E Beyzadeoglu [7,25,26], seem to publish the most promising articles on this subject in the last ten years. One of their last innovations was to position the medial portal for instrumentation a little more distal to patellar insertion.

Tsakotos [33] suggests an help from a needle as guide to injury location. In addition, a $70^{\circ}$ arthroscopy camera may favor the view of the tendon's posterior surface.

Therefore, all options exhibit advantages and disadvantages. What prevails in the end is the surgeon's technical experience, patient's satisfaction and return to sport at same previous level, which seems to be similar through both choices, with open surgery and arthroscopy.

\section{Conclusion}

Arthroscopy view is a valid option for surgery to correct an unresolved Osgood-Schlatter disease deformity. Functional results in

Citation: Marco Martins Lages and Max Rogerio Freitas Ramos. "Tibial Insertion of Patellar Tendon: Arthroscopic Approach". Acta Scientific Orthopaedics 4.3 (2021): 40-45. 


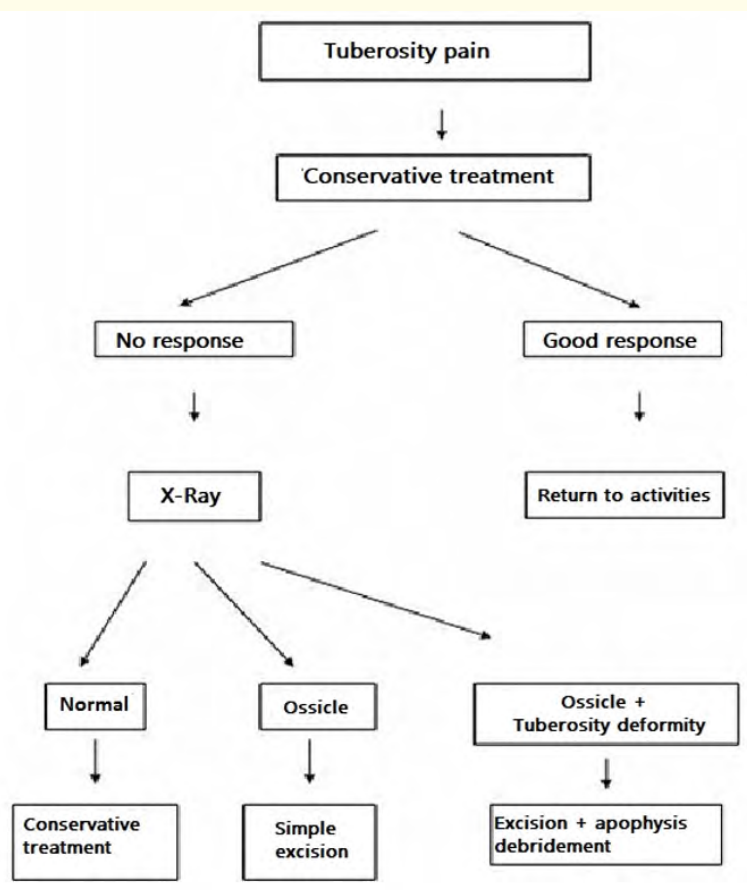

Figure 4 Unresolved Osgood-Schlatter treatment algorithm.

both choices, with open an arthroscopic surgery, demonstrate good results. Distal portals, between 2 and $4 \mathrm{~cm}$ of distal pole of patella provide the better arthroscopic view for this technique. Medial suprapatellar portal is a good option when an accessory portal is necessary.

\section{Bibliography}

1. Osgood RB. "Lesions of the tibial tubercle during adolescence". The Boston Medical and Surgical Journal 148 (1903): 114-117.

2. Schlatter C. "Verletzungen des schnabelformigen Fortsatzes der oberen Tibiaepiphyse". Beiträge zur klinischen Chirurgie 38 (1903): 8.

3. Kujala UM., et al. "Osgood-Schlatter disease in adolescente athletes. Retrospective study of incidence and duration". American Journal of Sports Medicine 13.4 (1985): 236-241.

4. Gholve PA., et al. "Osgood Schlatter Syndrome”. Current Opinion in Pediatrics 19.1 (2007): 44-50.
5. Deberardino TM., et al. "Arthroscopic treatment of unresolved Osgood-Schlatter lesions”. Arthroscopy 23.10 (2007): 1127.

6. Weiss JM., et al. "Surgical treatment of unresolved OsgoodSchlatter disease: ossicle ressection with tibial tubercleplasty". Journal of Pediatric Orthopaedics B 27.7 (2007): 844-847.

7. Beyzadeoglu T., et al. "Arthroscopic excision of an Ununited ossicle due to Osgood-Schlatter disease. Case Report". Arthroscopy 24.9 (2008): 1081-1083.

8. Klein W. "Endoscopy of the deep infrapatellar Bursa". Arthroscopy 12.1 (1996): 127-131.

9. Glynn MK and Regan BF. "Surgical treatment of OsgoodSchlatter's Disease". Journal of Pediatric Orthopaedics B 3.2 (1983): 216-219.

10. Nierenberg G., et al. "Surgical treatment of residual OsgoodSchlatter Disease in young adults: role of the mobile osseous fragmente". Orthopedics 34.3 (2011): 176.

11. Binazzi R., et al. "Surgical treatment of unresolved OsgoodSchlatter lesion". Clinical Orthopaedics and Related Research 289 (1993): 202-204.

12. Mital MA., et al. "The so-called unresolved Osgood-Schlatter lesion: a concept based on fifteen surgically treated lesions". Journal of Bone and Joint Surgery American 62.5 (1980): 732739.

13. Pihlajamäki HK., et al. "Long-term outcome after surgical treatment of unresolved Osgood-Schlatter Disease in Young men". Journal of Bone and Joint Surgery American 91.10 (2009): 2350-2358.

14. Pihlajamäki HK and Visuri TI. "Long-term outcome after surgical treatment of unresolved Osgood-Schlatter Disease in Young men - Surgical Technique". Journal of Bone and Joint Surgery American 92.258 (2010): 264.

15. Eun SS., et al. "Direct Bursoscopy Ossicle Ressection in Young and active patients with unresolved Osgood-Schlatter Disease". Arthroscopy 31.3 (2015): 416-421.

16. Figueiredo GC., et al. "Tratamento cirúrgico da dor crônica na lesão de Osgood-Schlatter: relato de dois casos". Revista Brasileira de Ortopedia 38.8 (2003): 491-496. 
17. Flowers MJ and Bhadreshwar DR. "Tibial tuberosity excision for symptomatic Osgood-Schlatter Disease". Journal of Pediatric Orthopaedics 15 (1995): 292-297.

18. Orava S., et al. "Results of surgical treatment of unresolved Osgood-Schlatter lesion". Annales Chirurgiae et Gynaecologiae 89 (2000): 298-302.

19. Hussain A and Hagroo GA. "Osgood-Schlatter Disease". Sports Exercise Injuries 2 (1996): 202-206.

20. Levine J and Kashyap S. "A new conservative treatment of Osgood-Schlatter disease". Clinical Orthopaedics and Related Research 158 (1981): 126-128.

21. Wysokinska A. "Rehabilitation after bursoscopy treatment of Osgood-Schlatter Disease - Preliminary report”. Ortopedia Traumatologia Rehabilitacja 9 (2007): 423-428.

22. El-Husseini TF and Abdelgawad AA. "Results of surgical treatment of unresolved osgood-schlatter disease in adults". The Journal of Knee Surgery 23 (2010): 103-107.

23. Lee YS., et al. "A case of arthroscopic removal of symptomatic ossicle associated with Osgood-Schlatter Disease in na athletic". The European Journal of Orthopaedic Surgery and Traumatology 21 (2011): 301-304.

24. Zhi-Yao L. "Arthroscopic excision of a huge ununited ossicle due to Osgood-Schlatter in an adult patient". Journal of Orthopaedic Case Reports 3.2 (2013): 4-7.

25. Circi E., et al. "Treatment of Osgood-Schlatter disease: review of literature". Muskuloskeletal Surgery 101. 3 (2017): 195-200.

26. Circi E and Beyzadeoglu T. "Results of arthroscopic treatment in unresolved Osgood-Schlatter disease in athletes". International Orthopedics 41.2 (2017): 351-356.

27. Lui TH., et al. "Endoscopic management of Osgood-Schlatter disease”. Arthroscopic Techniques 5.1 (2016): 121-125.

28. Schrouff I., et al. "How I treat... Osgood-Schlatter disease". Revue Medicale de Liege 70 (2015): 159-162.

29. Narayan N., et al. "Complete resolution of the symptoms of refractory Osgood-Schlatter disease following percutaneous fixation of the tibial tuberosity". British Medical Journal Case Reports (2015): bcr2014206734.

30. Cakmak S., et al. "Long-term outcome of Osgood-Schlatter disease: not always favourable". Rheumatology International 34 (2014): 135-136.

31. choi W and Jung K. "Intra-articular large ossicle associated to Osgood-Schlatter disease”. Cureus 10.7 (2018): e3008.

32. Pagenstert G., et al. "Reduction osteotomy of the prominent tibial tubercle after Osgood-Schlatter disease". Arthroscopy 33 (2017): 1551-1557.

33. Tsakotos G., et al. "Osgood-Schlatter lesion removed arthroscopically in an adult patient". Cureus 12.3 (2020): 7362.

\section{Assets from publication with us}

- Prompt Acknowledgement after receiving the article

- Thorough Double blinded peer review

- Rapid Publication

- Issue of Publication Certificate

- High visibility of your Published work

Website: www.actascientific.com/

Submit Article: www.actascientific.com/submission.php Email us: editor@actascientific.com

Contact us: +919182824667 